\title{
FEMINISMO, IZQUIERDAS,Y DERECHAS EN LA ERA DE LA REVOLUCIÓN NEOLIBERAL
}

\section{Feminism, Left and Rights in the Age of the Neoliberal Revolution}

\author{
Alessandro Caviglia* \\ Recepción: 05-1-2018 \\ Aceptación: 15-10-2018 \\ RESUMEN
}

El presente trabajo aborda la cuestión del feminismo de derecha y de izquierda en el contexto de la revolución neoliberal. Para ello, comienza a evaluar la discusión de si es posible un feminismo de derecha, para introducirla en un enfoque que permita verla a trasluz de la era neoliberal y colocar sobre mesa la distinción entre opresión y explotación.

En una segunda parte del trabajo se examina el debate actual sobre el feminismo y el abuso a través del debate generado por la Carta de las intelectuales francesas contra el movimiento \#metoo, la intervención de Mario Vargas Llosa respecto de una supuesta inquisición feminista en la literatura y en el arte, y los controversiales ataques de Camille Paglia contra el feminismo contemporáneo. El artículo termina articulando ambas partes explorando dos tensiones en el debate feminista.

\section{Palabras clave}

Feminismo, derecha, izquierda, revolución neoliberal, opresión, explotación.

\begin{abstract}
This paper addresses the issue of right and left feminism in the context of the neoliberal revolution. To do this, it begins by examining the discussion on whether a right-wing feminism is possible with the aim to frame the discussion in such a manner that allows us to see it in light of the neoliberal era and to bring to the table the distinction between oppression and exploitation. In a second part of the paper, the current debate on feminism and abuse is examined through the debate generated by the Charter of French intellectuals against the \#metoo movement, the intervention of Mario Vargas Llosa regarding a supposed feminist inquisition in literature and in art, and the controversial attacks of Camille Paglia against contemporary feminism. The paper ends by articulating both parts and exploring two tensions in the feminist debate.
\end{abstract}

\section{Keywords:}

Feminism, right, left, neoliberal revolution, oppression, exploitation.

* Filósofo. Profesor del Departamento de Teología de la Pontificia Universidad Católica del Perú y de la Carrera de Profesional de Filosofía de la Universidad Antonio Ruiz de Montoya: ascaviglia@yahooo.com 


\section{INTRODUCCIÓN}

A propósito del 8 de marzo del 2018, Día Internacional de la Mujer, Julia Mengolini escribió un breve artículo titulado "¿Puede el feminismo ser de derecha?" (Mengolini, 2018). Éste fue motivado por la frase de Malena Pichot según la cual "no se puede ser de derecha y feminista" ${ }^{1}$ y que terminó generando controversia por las redes sociales, especialmente en Argentina. La posición de Pichot y Mengolini puede dar pie a un ejercicio interesante para echar luces sobre la pregunta de si existe o si es posible un feminismo de derechas en la actualidad. Otros tres aspectos interesantes respecto del tema en torno al feminismo son a) constatar de qué manera muchas mujeres han estado votando en las elecciones políticas de muchos países democráticos por candidatos que representan lo opuesto a sus intereses, b) el fenómeno suscitado en los últimos años en torno al incremento del feminicidio, especialmente en América Latina y la respuesta frente a este, bajo la forma de las marchas $\mathrm{Ni}$ una menos y c) los movimientos \#MeToo o \#balancetonporc, movimientos que han generado reacciones directas o indirectas como la denominada Carta Denueve (o también llamado Manifiesto de las intelectuales francesas contra el \# MeToo) ${ }^{2}$, la intervención de Mario Vargas Llosa sobre el feminismo y la literatura, y las también polémicas posiciones de Camille Paglia en torno al género y al feminismo.
Ciertamente, los cuatro puntos que he mencionado conectan cuestiones de carácter político y económico con cuestiones de carácter moral. La crítica de Deneuve al supuesto nuevo puritanismo que el feminismo está imponiendo a causa de las acusaciones hacia los acosadores puede ser vista como un aspecto más de tinte moral en torno a la cuestión feminista. Pero la posición de Paglia pretende articular ese nuevo puritanismo con una actitud de que sería la de las mujeres de clase media alta, que querrían seguir realizando su liberación sexual pero en un mundo sin peligros y controlado como "las salas de estar de sus casas" (dicho en los términos de la polémica intelectual ítalo-estadounidense), mientras que las mujeres de "la clase trabajadora" no tendrían esa pretensión, sino que se encontrarían dispuestas a asumir los riesgos que la sociedad actual implica y aprenderían a defenderse por sí mismas. Lo que Camille Paglia realiza con esa forma de argumentación es establecer el vaso comunicante entre los aspectos más morales con los aspectos más económicos y políticos en torno a la cuestión del feminismo, pero ciertamente de manera polémica.

Dividiré el presente trabajo en dos secciones. En la primera me concentraré en los aspectos que tienen un componente de índole político y económico, mientras que, en la segunda, en los aspectos más cargados de cuestiones morales, pero sin los vasos comunicantes entre ambos aspectos. Ahora bien, una de las

1 https://www.ratingcero.com/notas/3064056-video-malena-pichot-dijo-que-no-se-puede-ser-derecha-y-feminista-y-estallo-la-polemica.

2 https://www.infobae.com/america/mundo/2018/01/09/el-manifiesto-completo-de-las-intelectuales-francesas-contra-el-metoo/. El grupo de estas intelectuales fue encabezado por Catherine Deneuve y Catherine Millet. La respuesta a dicho manifiesto no se hizo esperar y vino de parte de un grupo de feministas liderado por Caroline de Haas https://www.francetvinfo.fr/societe/droits-des-femmes/tribune-les-porcs-et-leurs-allie-e-s-ont-raison-de-sinquieter-caroline-de-haas-et-des-militantes-feministesrepondent-a-la-tribune-publiee-dans-le-monde 2553497.html. En este conjunto de intervenciones se pueden detectar dos temas claramente diferenciados. El primero versa sobre la naturaleza política del feminismo, es decir, si es de izquierda o de derecha. El segundo, en cambio, tiene que ver el carácter de los movimientos como \#MeToo o \#balancetonporc. 
carencias en muchos de los discursos que se encuentran en el debate en torno al feminismo contemporáneo es la ceguera frente al marco político y económico articulado a partir de la llamada "revolución neoliberal" (Honneth, 2009). Es por esa razón que realizaremos un análisis de los discursos feministas y antifeministas más relevante utilizando como espejo el contexto neoliberal en el que las mujeres y los varones tienen que definir y redefinir sus relaciones y su autocomprensión.

\section{1.- Feminismo, política y economía en la era de la revolución neoliberal}

Para poder abordar los aspectos de índole político y económico es necesario que situemos el contexto político, social y económico en el que nos encontramos. Desde la década de los 90 nos encontramos dentro del despliegue de la denominada "revolución neoliberal". Las ideas neoliberales se fueron gestando a raíz de la crisis de 1929 y fueron adquiriendo su contextura básica en el conocido Coloquio Lippmann, desarrollado en París en agosto de 1938 (Foucault, 2008), prácticamente en las vísperas de la II Guerra Mundial. Sin embargo, las políticas neoliberales no se implementan ni antes ni después de la guerra, sino a partir de los 70. Después de la guerra se implementan tanto en Estados Unidos como en Europa políticas económicas de raigambre keynesianas y fordistas que generaron un capitalismo dirigido desde el Estado (y, en América Latina dichas políticas tuvieron su expresión en las políticas desarrollistas emprendidas por los Estados). Estas políticas constituyeron lo que se conoce como "la era dorada del capitalismo" donde se instauró en Estados Unidos y en Europa el llamado "Estado de bienestar".
Sin embargo, a raíz de la llamada crisis del petróleo suscitada a fines de la década de los 60 y principios de los 70 la economía del "Estado de bienestar" entra en crisis debido al alza del precio del crudo y los Estados comienzan a implementar políticas neoliberales. Primero se implementaron en 1973 en Chile durante el gobierno de Pinochet, después entre 1979 y 1980 en Inglaterra con Margaret Thatcher y en Estados Unidos con Ronald Reagan. Durante la década de los 90, a raíz de la Caída del Muro de Berlín y los avances tecnológicos y en la comunicación, se expande a lo largo de todo el mundo, constituyendo un mercado mundial.

Durante las administraciones de Bill Clinton en Estado Unidos y de Tony Blair en Inglaterra el neoliberalismo adquiere una doble mutación. La primera es que gira hacia un capitalismo financierizado, en el cual instituciones como los bancos y las bolsas van a tener un mayor peso que las industrias. En muchas partes del mundo las industrias van a ser desmanteladas porque se le va a dar un mayor peso a la economía especulativa. El segundo giro lo va a constituir la articulación de lo que hoy en día se conoce como "neoliberalismo progresista", que incorpora a la dinámica de acumulación neoliberal las políticas identitarias centrales del progresismo académico desarrolladas durante la década de los 90, a saber, la política del multiculturalismo, la política feminista y la política racial, políticas que se centran en el reconocimiento de la identidad y que dejan fuera el problema de la redistribución socioeconómica.

El ascenso del populismo tanto en Estados Unidos, Europa y en América Latina va como punto de ataque a esta forma compleja de neoliberalismo 
articulado financieramente y centrado en las políticas de la identidad. Es por ello por lo que los populismos (tanto de derecha como de izquierda) que comienzan a tener fuerza desde el 2014 reivindican los intereses de la clase trabajadora en contra de las políticas de austeridad económica (como es el caso de Alternativa para Alemania, el Movimiento Cinco Estrellas en Italia, Podemos en España y Siryza en Grecia) y atacan las políticas identitarias por considerarlas encubridoras de la desigualdad y representantes de una élite tanto política como intelectual. Es de esta manera que Donald Trump ataca al feminismo, al multiculturalismo y a la política racial, mientras que Bernie Sanders (y ciertamente, también Trump y los demás populistas) afilan sus ataques contra la mala distribución socioeconómica y reivindican los intereses de la clase trabajadora.

En el caso de América Latina, los populismos atacan tanto a la élite intelectual como al sistema económico en diferente medida. En Venezuela el chavismo la emprendió contra el sistema económico (con resultados nefastos), lo mismo sucedió en Ecuador y en Bolivia, países en los que se fortaleció una élite intelectual que tomaba distancia de las políticas de la identidad y se conectaba más con ideales marxistas o neomarxistas. En el caso del Perú, el Movimiento Fujimorista arremetió contra la élite intelectual y las políticas identitarias, refiriéndose a sus representantes intelectuales y políticos con el apelativo de "caviares". De esta manera, el populismo tanto en Estados Unidos, en Europa como en América Latina dirige su ataque contra el sistema económico (atacando especialmente las políticas de integración económica -exigiendo el "cierres de las fronteras"- - y de la justicia -exigiendo la salida del sistema internacional de Derechos Humanos-).

\section{1.- Feminismo, opresión y explotación}

La filósofa estadounidense, Nancy Fraser (Fraser, 2015) ha aclarado suficientemente de qué manera el llamado "Feminismo de la segunda ola", que se inicia después de la Segunda Guerra Mundial y se prolonga hasta nuestros días, ha tenido dos ejes de cuestionamiento en la mira: la opresión en el hogar y la explotación en el trabajo. Al mismo tiempo, las sociedades pasaron del "capitalismo organizado desde el Estado" (en Estados Unidos y en Europa) y los "Estados desarrollistas" (en América Latina) a la expansión global de las reformas neoliberales a partir de los años 90. En ese trance, el feminismo giró de una plataforma que buscaba mayores reivindicaciones económicas para las mujeres (y la posibilidad de ser insertadas en el mercado laboral con un sueldo justo y paritario frente al sueldo de los varones) a las exigencias del reconocimiento de la identidad de las mujeres. De tal manera que las luchas feministas más influyentes pasaron del problema de la redistribución socioeconómica al problema del reconocimiento de la identidad. Con ello se produjo una domesticación de las exigencias feministas por parte del proyecto político neoliberal.

Así, desde los 90 en adelante, las exigencias de reconocimiento de la diferencia entre los varones y las mujeres dejó en un segundo plano las reivindicaciones económicas. Y es que en el tránsito a la década de los 90, la implementación de las reformas neoliberales, cancelaron el llamado "Estado de bienestar" que caracterizó a la época de oro del capitalismo (en el que los Estados ejercían control de los mercados) y lo sustituyó por el "Estado de precariedad" y por una época del capitalismo en el cual los mercados desembocados ejercen el control de los Estados. 
En este nuevo contexto, las mujeres experimentan no sólo la opresión por parte del varón dentro del hogar (que estaba presente en el capitalismo de posguerra) sino que a ello se suma la sobreexplotación en el trabajo. En el contexto neoliberal esta sobreexplotación se incrementa con la auto-explotación que sufren tanto varones y mujeres. Esta se caracteriza por la exigencia que las personas se autoimponen de capacitarse constantemente a fin de ser cada vez más competitivas en el mundo del trabajo. Es lo que se conoce como "incremento de capital humano" Así, el contexto neoliberal modifica las exigencias de los movimientos feministas, debido a las transformaciones socioeconómicas propias del Estado de precariedad y por la incorporación de los estudios culturales en el imaginario de las luchas feministas. Un ejemplo de ello lo representan los trabajos de Judith Butler, quien se centra en las cuestiones sociales y simbólicas del feminismo. Pero como bien ha señalado Martha Nussbaum (Nussbaum, 2000 b), el enfoque de Butler pierde de vista por completo la perspectiva política que se encuentra detrás de la opresión y la explotación contra la mujer. Ciertamente, la crítica que Fraser haría a Nussbaum es que su análisis político pierde de vista la conexión entre la política y el modelo económico en la era neoliberal.

De esta manera, lo que exigen es el reconocimiento de las mujeres en su identidad como mujeres (en vez de girar hacia la exigencia de reconocimiento de status) y la posibilidad de llegar a los mismos ascensos que los varones en las corporaciones trasnacionales. Con ello, queda fuera de las exigencias feministas la necesidad de transformar el modelo económico neoliberal y el cuestionamiento de la desigualdad en condiciones de vida que existe entre mujer y mujer. En el nuevo contexto neoliberal, las mujeres que han accedido a la mejor educación pueden aspirar ocupar cargos de alta dirección en las grandes corporaciones, mientras que las que no han podido hacerlo carecen muchas oportunidades laborales con ingresos sustantivos. Incluso las primeras contratan a las segundas para que se hagan cargo de sus casas, de sus hijos o de sus padres de la tercera edad (esto es lo que ocurre a menudo en Estados Unidos y en Europa, donde las mujeres migrantes se hacen cargo de los hijos o de los padres de mujeres que son ejecutivas de empresas trasnacionales, recibiendo bajos ingresos por su trabajo). Las feministas, centradas en las cuestiones de identidad y de diferencia, dejan sin cuestionamiento el modelo neoliberal que termina radicalizando tanto la opresión en el hogar como la explotación en el trabajo.

\section{2.- ¿Es el feminismo sólo de izquierda?}

El argumento de Mengolini señala categóricamente que no es posible un feminismo de derecha. Este se desarrolla por pasos. El primero es señalando que las manifestaciones Ni una menos pueden terminar aglutinando personas de diferentes sectores, tanto de derecha como de izquierda, pero el hecho de que alguien participe de ellas no basta para que se esté apoyando la causa feminista en la realidad. Participar en dichas manifestaciones representa un mínimo que sólo exige que no se asesine a las mujeres por el hecho de ser mujeres. El segundo paso del argumento es señalar que el feminismo es aquel modo de entender que permite ver con claridad que el asesinado de una mujer, por el hecho de ser mujer, es el resultado de un sistema patriarcal en el que el "un hombre mata a una mujer porque cree que es dueño de la vida de esa mujer". De manera que se trataría de "una extensión del patriarcado" 
y "la expresión final de dicho sistema". Así, el patriarcado sería un sistema que incorpora un mecanismo que tiene como punto final el asesinato de una mujer por simple hecho de serlo.

El tercer paso el argumento consiste en señalar que la derecha no ve la realidad que constituye el patriarcado, porque hacerlo sería colocar sobre la mesa de debate el problema de la igualdad. Esto es así porque el patriarcado se funda en la desigualdad entre hombres y mujeres (que, aunque la autora no especifica, podríamos suponer que se refiere a la desigualdad económica y la desigualdad de estatus entre hombres y mujeres). El cuarto paso consiste en señalar que la derecha se reúsa a poner en discusión la cuestión de la igualdad porque ello supondría cuestionar tanto el sistema legal, el sistema social y el sistema económico. En consecuencia, no es posible un feminismo de derecha, porque desde sus inicios el feminismo se ha planteado atacar la cuestión de la desigualdad legal, de estatus y económica entre hombres y mujeres.

El argumento parece claro y convincente. Pero no tiene en cuenta el contexto actual en el que el feminismo se encuentra inserto, contexto marcado por la revolución neoliberal y la consecuente distinción entre opresión en el hogar y explotación en el trabajo. Cuando la autora argentina señala lo que el feminismo debe de plantease es transformar la sociedad, ¿̇a qué se está refiriendo?: ¿a transformar las relaciones entre mujeres y varones dentro del hogar?, ¿a superar la desigualdad remunerativa entre mujeres y varones en el mundo del trabajo? (pero dentro del esquema político y socioeconómico vigentes después de la implementación de las reformas neoliberales) o ctransformar el modelo político-económico impuesto tras la revolución neoliberal? Por no tener claridad respecto del trasfondo en el que el feminismo de la segunda ola se desarrolla después del fin de la "época de oro del capitalismo", el análisis de la autora pierde perspectiva.

\section{3.- Feminismo de derechas y feminismo de izquierdas}

Muy en contra de lo que Mengolini y Pichot sostienen, sí existe un feminismo de derecha. Alguien que expresa con claridad las ideas de una forma moderada de feminismo de derecha es la abogada y periodista Rosa María Palacios. Ella sostiene que lo que necesitan las mujeres es mayor educación para que puedan tener independencia económica y puedan salir del círculo de opresión, abuso y muerte que se produce al interior del seno familiar. Ciertamente, el argumento de Palacios alcanza los derechos laborales y el derecho al voto, pero para ella el problema no es que el sistema económico y social tenga que ser cambiado, sino que dentro del mismo sistema imperante las mujeres no acceden a los mismos derechos a los que acceden los varones. Es por ello por lo que, para la abogada y periodista, la lucha de las mujeres debe tener como punto central la educación para que así puedan acceder a los derechos disponibles por el sistema neoliberal. Ese tipo de feminismo se encuentra dirigido a atacar la opresión, pero deja sin cuestionamiento el sistema económico neoliberal que genera la explotación en el trabajo y que se expresan en altas horas de trabajos, bajos sueldos y precarias condiciones.

Una forma radicalizada del feminismo de derecha es el que se articula en torno a la retórica de la supuesta "libertad de elección". Se trata de quienes reivindican el derecho a elegir de las mujeres si quieren mantenerse dentro de la opresión 
y desean elegir una vida de amas de casa sometidas al varón ${ }^{3}$. Ciertamente, la libertad de elegir es importante y constituye una forma significativa de lo que llamamos autonomía, pero bajo esta figura, la libertad de elegir termina anulando la autonomía porque lo que se estaría eligiendo es ceder toda la autonomía de la persona a otro. De esta manera, el contrasentido de este feminismo consiste en que en nombre de la libertad de elección la mujer estaría entregándose a la dominación del varón. En muchos casos, este argumento, que en apariencia es feminista, resulta ser el de personas que se encuentran conectadas con modos conservadores de religión. Así como el creyente más conservador decide someter su voluntad a los mandatos de Dios y a las autoridades que supuestamente lo representan, del mismo modo deciden someterse a la voluntad varón.

La primera forma de feminismo de derecha se centra en la defensa de la autonomía y de los derechos de las mujeres. La idea de dotarlas de educación se dirige a que puedan ser independientes y puedan reivindicar sus derechos. De esta manera, esta forma de feminismo entiende que existe una conexión interna entre la autonomía moral de la persona y la reivindicación de sus derechos al interior de una sociedad democrática basada en principios constitucionales sólidos. En cambio, la segunda forma de feminismo de derecha se entronca en lo que con claridad Ronald Reyes denomina populismo. De acuerdo con la observación de Reyes, la estrategia del populismo consiste en desconectar la libertad de elección de la autonomía moral de las personas. Así, en la segunda forma de feminismo de derechas se expresa el populismo que tiene como objetivo hacer saltar los derechos constitucionales anclados en la autonomía moral de las personas y reemplazarlos por una democracia plebiscitaria que puede legitimar la renuncia a la autonomía moral en nombre del derecho a la libertad de elección.

El feminismo de izquierda, en cambio, no sólo se centra en el cuestionamiento de la opresión, sino también en el de la explotación. Pero aquí hay dos posibles opciones. La primera es que las feministas exijan que las mujeres ganen lo mismo que los varones en el mercado laboral, mientras que la segunda en que las mujeres se unan a los varones y los demás grupos que se encuentran luchando en contra del sistema político y económico del neoliberalismo. El feminismo que no cuestiona el sistema neoliberal se parece al feminismo de derechas moderado en que no cuestiona el sistema de explotación, pero sí la opresión. Pero mientras que el feminismo de derecha moderado se centra en la importancia de la educación para que las mujeres puedan acceder al mercado laboral en condiciones igualitarias a los varones, el feminismo de izquierda que no cuestiona el neoliberalismo (como es el caso de Mengolini y Pichot) sino que cuestiona la cultura androcéntrica que articula la sociedad. Para ellas, la razón por la cual las mujeres no pueden acceder a mejores condiciones socioeconómicas se encuentra en una cuestión cultural (la cultura patriarcal) y no en una cuestión de organización de la estructura política y económica de la sociedad. De esta manera, no se ve con claridad las diferencias más importantes entre este feminismo de izquierda y el feminismo de derecha moderado, tal

3 Véase, al respecto: https://pareja.enfemenino.com/foro/quiero-ser-ama-de-casa-fd1723128. Y también: https://lamenteesmaravillosa.com/quiero-madre-ama-casa/. 
como lo defiende Rosa María Palacios. Una variante de este feminismo de izquierda lo representa Martha Nussbaum (Nussbaum, 1999, Nussbaum, 2000). Su feminismo, centrado en el enfoque de las capacidades desarrollado por ella y por Amartya Sen y en la idea de Desarrollo Humano plantea una forma de entender el desarrollo de las mujeres, pero no tiene ni un análisis del neoliberalismo imperante ni tampoco críticas al mismo. De esta manera, puede plantear una crítica a la política que no permite que las mujeres puedan gozar de los mismos derechos que los varones, pero no logra articular una crítica al proyecto político neoliberal imperante desde la década de los 90.

Nancy Fraser, por su parte, es una de las representantes de un feminismo de izquierda que establece una conexión clara entre la lucha feminista y el cuestionamiento del neoliberalismo imperante. Esta perspectiva permite ver con claridad que el problema de las mujeres no es solo que no acceden a los mismos derechos que las mujeres, sino que el sistema político y económico de la era de la sociedad de la precariedad no permite poner a disposición de las mujeres los derechos adecuados para conseguir reconocimiento en tanto que mujeres, al mismo tiempo que, distribución económica justa y paridad participativa en un sistema político capaz de someter al Estado y al mercado al control democrático.

El neoliberalismo imperante, más bien, lo que hace es exigir a las mujeres a que se auto-exploten a sí mismas para que sean más competitivas en el mercado, desarrollando al máximo su "capital humano". Es por eso por lo que la educación no basta, puesto que en la era de la revolución neoliberal, las mujeres suficientemente educadas terminan entrampadas en la carrera por ocupar los altos cargos de dirección de empresas que se articulan por los imperativos de los mercados desembocados. De manera que si las mujeres pueden romper el llamado "techo de cristal" (que les impide acceder a los altos cargos en las corporaciones trasnacionales) lo que no pueden es quebrar lo que podríamos denominar el "techo de hierro" representado por las continuamente ajustadas reformas neoliberales que terminan por exigir a las mujeres a someterse a la opresión de no tener una realización personal fuera del trabajo y a un doble nivel de explotación. El primer nivel lo constituye las exigencias del neoliberalismo de someterse a un constante incremento de la desigualdad; mientras que el segundo nivel lo constituye la exigencia de las mujeres más educadas de autoexplotación para mantenerse en el top de la competencia neoliberal.

La perspectiva de Fraser permite percibir además que la exigencia de productividad sobre las empresas que viene del proyecto político neoliberal hace que la desigualdad se incremente cada vez más. De esta manera, el neoliberalismo no solo hace que la desigualdad entre varones y mujeres se incremente, sino también entre varón y varón, y entre mujer y mujer. De manera que el despegue en la competencia que tienen las mujeres más educadas en las grandes corporaciones es posible porque las mujeres de menor educación y menores recursos hacen los trabajos más humildes y que tienen menor remuneración. De esta manera, el neoliberalismo no sólo enfrenta a las mujeres contra los varones, sino también a las mujeres entre sí, puesto que las mejoras en las condiciones de vidas de unas se logra gracias al constante empeoramiento de las condiciones de vida de otras. 


\section{4. - ¿Por qué muchas mujeres defienden posiciones políticas y votan por candidatos que van en contra de sus propios intereses?}

Fraser presenta con claridad por qué razones las mujeres norteamericanas votaron en contra de sus propios intereses, ya sea por George Bush en el 2004 como por Hillary Clinton el 2008. Así también, tiene una explicación elocuente del triunfo de Donald Trump en el 2016. Ella señala que "las feministas se quedaron sorprendidas al descubrir que, mientras ellas habían estado discutiendo sobre esencialismo, una non sancta alianza entre defensores del libre mercado y cristianos fundamentalistas se habían adueñado del país" (Fraser, 2008, 198). De tal manera que mientras la derecha representada por Bush en el 2004, de una parte se enarbolaba las banderas de las llamada "guerra contra el terrorismo" y la de los "valores familiares (Fraser, 2008, 198) (especialmente el cuestionamiento al derecho al aborto y al matrimonio entre personas del mismo sexo), y de otra parte representaba la continuidad del modelo neoliberal que incrementa la riqueza de las corporaciones y los sectores más adinerados, eliminando el impuesto sobre sucesiones, disminuyendo los tipos impositivos de la riqueza y obligando a la clase trabajadora que incremente mucho más su contribución al presupuesto nacional (Fraser, 2008, 198-199).

La retórica de la guerra contra el terrorismo incluía que, ante la amenaza, los y las votantes deberían elegir a un presidente que represente la virilidad (con la fortaleza y la firmeza que supuestamente ello implica) y no a uno que pudiese representar la feminidad (con la actitud dubitativa que supuestamente implica). Dicha retórica exigía, en consecuencia, que los y las estadounidenses votasen por George Bush en vez de John Kerry. Pero la consecuencia de ello era que las mujeres votarían un sistema que incrementaba el "estado de precariedad e inseguridad" que se había instalado en la sociedad norteamericana desde la revolución neoliberal y que se traducían en la "reducción de impuestos a las empresas y a la clase rica, la disminución del bienestar social y de la protección al consumidor, salarios muy bajos y empleo precario" (Fraser, 2008, 200). Estas políticas obligaron a que las mujeres tuviesen no sólo que salir a trabajar en el mercado neoliberal, sino a verse obligadas al pluriempleo para poder mantener la economía familiar, a la vez de tener que hacerse cargo de la casa.

Sin embargo, la retórica de la lucha contra el terrorismo y de la defensa de los valores familiares condujo a muchas mujeres a elegir a Bush. Además, la retórica de la derecha consistía en desautorizar a las feministas, quienes eran retratadas como mujeres de clase media alta y académicas que se contraponían a los intereses de las mujeres de la clase trabajadora. Así (tal como la retórica de la derecha pintaba el panorama), mientras que las feministas atacaban los "valores familiares" (como la condena al aborto y la condena al matrimonio igualitario), las mujeres de la clase trabajadora se identificaban con dichos valores y se veían a sí mismas como amas de casa por más que ya se desarrollaban en el mundo del trabajo articulado a la medida de las reformas neoliberales. Al mismo tiempo, muchos grupos feministas habían abandonado ya la gramática de la lucha distributiva y había abrazado la del reconocimiento, a la que dotaron (de manera equivocada) de un giro hacia la identidad y hacia el esencialismo en vez de un giro hacia la reivindicación del status. 
El cristianismo evangélico fundamentalista aportó su cuota de apoyo a la derecha conservadora y neoliberal al convertirse en un lugar de acogida de las mujeres en la era de "la sociedad de la precariedad". La retórica de ese tipo de cristianismo decía lo siguiente: "Eres una pecadora, vas a fracasar, puedes perder tu trabajo, es posible que te des a la bebida, a lo mejor tienes una aventura, quizás tu marido te abandone, tus hijos pueden ser drogadictos. Pero Dios te ama a pesar de todo, y tu Iglesia siempre te va a acoger" (Fraser, 2008, 202-203). El objetivo de este discurso era que las mujeres acepten la situación a la par de prepararlas para los momentos difíciles. De esta manera, el cristianismo de derecha (que era defensor de los valores familiares tradicionales) colaboraba con que las mujeres acepten el sistema neoliberal y voten por candidatos que se oponen a sus intereses tanto sociales, económicos como culturales. Además, en algunos casos, el cristianismo de derecha se opone a la planificación familiar y al control del propio cuerpo de parte de la mujer. La retórica del cristianismo de derecha incorpora la idea de que es la Iglesia la que debe de tener el control del cuerpo de la mujer, combatiendo los anticonceptivos y atacando tanto cualquier tipo de aborto como el disfrute del sexo recreativo tanto fuera como dentro del matrimonio.

En el Perú, la estrategia ha sido la misma. La derecha política neoliberal se ha aliado, desde los 90, con la derecha católica y cristiana. Las estrategias de dirigir el voto de las mujeres hacia la derecha radical que el fujimorismo representa, desautorizar a las feministas (tildándolas de "caviares", es decir, académicas de clase media alta) y la política del control del cuerpo (hasta llegar a la política de las esterizaciones forzadas); todas esas estrategias responde a los imperativos que provienen de la alianza entre el cristianismo y el catolicismo de derecha con los portadores del neoliberalismo radicalizado que el fujimorismo representa por medio de leyes como la llamada "ley de esclavitud juvenil".

La estrategia de la derecha no se dirige a que las mujeres voten por un varón, sino que lo hagan por quien representa lo viril. En las primarias demócratas del 2008 las mujeres de la clase trabajadora norteamericana aceptaron la exigencia de la derecha de votar por Hillary Clinton y no por Barack Obama. Si bien Clinton es mujer, ella representa lo varonil (con la fortaleza y la determinación que supuestamente ello implica) mientras que Obama, siendo varón, era representado como lo femenino (con la indecisión y el temor que supuestamente ello implica). Lo mismo se puede ver en el Perú en la comparación entre Keiko y Kenji Fujimori. Mientras que la primera representa lo varonil, el segundo representa lo femenino. La retórica de la derecha conservadora en el Perú para con las mujeres sigue siendo la misma que la que se ha impuesto en todo también en EE.UU. y en Europa: las mujeres deben votar por quien representa al varón, en contra de sus intereses. Durante la contienda electoral estadounidense del 2016 el esquema se reprodujo, con ciertas variantes. En las primarias norteamericanas, Clinton representaba al varón defendiendo los intereses del mercado y Bernie Sanders representaba a la mujer defendiendo los intereses de la clase trabajadora. Pero en la contienda presidencial Donald Trump representaba más al varón que Clinton. Lo interesante de ese proceso fue que tanto Trump como Sanders representaban los intereses de la clase trabajadora, pero mientras que el primero lo hacía representando también el saltar por encima de la democracia (y colocándose 
en contra de las feministas, los defensores del multiculturalismo y la política racial - es decir, poniéndose en contra de las políticas del reconocimiento), Sanders representaba a la clase trabajadora pero también la defensa de la democracia y las políticas del reconocimiento). De manera que, si la elección presidencial terminaba siendo entre Trump y Sanders, muchas mujeres de la clase trabajadora posiblemente habrían apoyado a Trump porque él representa lo masculino y ataca la política feminista.

\section{2.- Feminismo y moral en el contexto de la revolución neoliberal}

A raíz de casos como el de Harvey Weinstein, se han suscitado una serie de denuncias bajo el apoyo de grupos como \#metoo y \#balancetonporc, como otros grupos feministas. Pero la actividad de estos grupos y de grupos anteriores han recibido respuestas que resultan ser controversiales. Tres de estas respuestas fueron a) la Carta Deneuve, b) el artículo de Mario Vargas Llosa y c) la posición de Camille Paglia. En lo que sigue analizaremos las tres posiciones y mostrar los bemoles de dichas posiciones.

\section{1.- La Carta Deneuve}

La llamada Carta o Manifiesto Deneuve (Deneuve, 2017) señala lo siguiente. En primer lugar, que, si bien la violación es un crimen, el coqueteo insistente y torpe no lo es, y tampoco la galantería es una agresión machista. Pero, sin embargo, la "liberación de la palabra se convierte hoy en su opuesto. iNos ordenan hablar, a silenciar lo que enoja, y aquellos que se niegan a cumplir con tales órdenes se consideran traidores, cómplices!", señalan las firmantes. De tal manera que se estaría instalando un puritanismo que resultaría conservador. De modo que "es una característica del puritanismo tomar prestado, en nombre de un llamado bien general, los argumentos de la protección de la mujer y su emancipación para vincularlas a un estado de víctimas eternas, pobres pequeñas cosas bajo la influencia de demoniacos machistas, como en los tiempos de las brujerías". Y sigue afirmando que el movimiento "\#metoo ha provocado en la prensa y en las redes sociales una campaña de denuncias de personas que, sin tener la oportunidad de responder o defenderse, fueron puestos exactamente en el mismo nivel que los delincuentes sexuales".

Entonces el Manifiesto denuncia un presunto puritanismo enemigo de la libertad sexual de las mismas mujeres, que las convertiría en infantes y no en adultas. De esta manera, se señala que "esta fiebre por enviar a los "cerdos" al matadero, lejos de ayudar a las mujeres a empoderarse, en realidad sirve a los intereses de los enemigos de la libertad sexual, los extremistas religiosos, los peores reaccionarios y los que creen - en nombre de una concepción sustantiva de la moralidad buena y victoriana - que las mujeres son seres "separados", niñas con una cara de adulto, que exigen protección".

El siguiente paso de la Carta consiste en defender el presunto "derecho a importunar". Para ello se hace una analogía con el arte. Se señala que "el filósofo Ruwer Ogien defendió una libertad de ofensa indispensable para la creación artística. De la misma manera defenderemos una libertad para importunar, indispensable para la libertad sexual". De tal manera que en nombre de la libertad para importunar las firmantes terminan su manifiesto con esta frase: "como mujeres, no nos reconocemos en este feminismo que ... toma el rostro del odio hacia los hombres y 
la sexualidad". De manera que, de acuerdo a las firmantes del manifiesto, la actitud del colectivo \#metoo lo que expresan es el odio a los hombres y a la sexualidad.

Más allá de los errores o excesos que se pudiesen cometer al denunciar a algunas personas inocentes de acosadores, lo que el Manifiesto deja fuera de visión es que la organización de la sociedad no coloca a los hombres y a las mujeres en condiciones de igualdad, sino que debilita la posición de las mujeres. Es la misma sociedad androcéntrica la que procura constantemente disminuir la posición de las mujeres y tornarlas expuestas a peligros a los que los varones no se encuentran. También es posible que en el afán de denunciar a los acosadores se podrían cometer excesos contra personas inocentes, pero lo cierto es que invitar a las mujeres a que denuncien a sus acosadores no necesariamente tiene que consolidar una moralidad puritana y conservadora. La libertad de denunciar a los acosadores no necesariamente conduce a una libertad sexual. Lo que tiene que lograrse, más bien, es romper el esquema social androcéntrico para lograr dotar tanto a las relaciones entre varones y mujeres de mayor libertad.

Ahora bien, el análisis del Manifiesto Deneuve nos conduce a dos caminos interesantes. De un lado se encuentra la asociación entre la libertad para importunar con la libertad para ofender a través de la producción artística. Por este camino se dirige el errático artículo de Mario Vargas Llosa. Por otro lado, se encuentra la diferencia entre las mujeres de clase media alta y las mujeres de la clase trabajadora, que se encuentra completamente ausente en el Manifiesto y que es explotado por Camille Paglia para fortalecer un antifeminismo de derecha sofisticado.

\section{2.- Mario Vargas Llosa, literatura y feminismo}

En un artículo titulado "Nuevas inquisiciones" (Vargas Llosa, 2018) en el diario El País, el Nobel Mario Vargas Llosa señaló que el feminismo se ha convertido en un inquisidor de la literatura. El escritor señala que "ahora el más resuelto enemigo de la literatura, que pretende descontaminarla de machismo, prejuicios múltiples e inmoralidades, es el feminismo. No todas las feministas, desde luego, pero sí las más radicales, y tras ellas, amplios sectores que, paralizados por el temor de ser considerados reaccionarios, ultras y falócratas, apoyan abiertamente esta ofensiva antiliteraria y anticultural" (Vargas Llosa, 2018).

Esta afirmación, que constituye el corazón de su argumentación, está basada en una falacia de generalización que, aunque señala que lo que está describiendo es la actitud de un grupo de feministas más radicales, termina por extenderlo al feminismo en general. De hecho, menciona el caso de la actitud contra Neruda en determinadas escuelas o la actitud contra la novela Lolita de Nabokov. En su afán defensor de la libertad literaria, el escritor hispano peruano parece olvidar algo que sabe muy bien, a saber, la receptividad de una obra de arte depende de la subjetividad de quien la lee o la contemple. De hecho, existen testimonios de mujeres que señalan que la lectura de la Novela de Nabokov fue sumamente liberadora. Un ejemplo de ello lo constituye el artículo de Bindu Bansinath (Bansinath, 2018). Y, estoy seguro, que muchas feministas saben eso. Muchas de ellas saben que la literatura es liberadora y apoyarían las ideas de George Bataille que nuestro Premio Nobel parafrasea de la siguiente manera: "todo aquello que debe ser reprimido para hacer posible la sociedad —los instintos 
destructivos, "el mal" - desaparece sólo en la superficie de la vida, no detrás ni debajo de ella, y que, desde allí, puja para salir a la superficie y reintegrarse a la existencia. ¿De qué manera lo consigue? A través de un intermediario: la literatura" (Bansinath, 2018).

Ahora bien, existen dos razones por la cuales una persona o un grupo podría pretender censurar una obra de arte: por su contenido y por su autor. Si alguien quiere censurar la novela Lolita lo sería porque considera que su contenido es inapropiado, por considerar que cuenta una historia degradante de una joven mujer. Otra cosa es querer censurar la poesía de Neruda o la retrospectiva de Roman Polanski por la conducta de los artistas respecto de las mujeres. En ambos casos, intentar censurar las obras de arte no resulta correcto ni democrático. Tengo la seguridad que la mayoría de los feministas no están de acuerdo con esas censuras. Una cosa es censurar la conducta de Polanski y llevarla ante los tribunales y otra es censurar sus películas. Reconocer esa diferencia es dar muestras de sensatez. Resulta claro que Vargas Llosa está cargando contra un enemigo que no existe o que es reducido o marginal.

\section{3.- La revancha conservadora contra el feminismo de Camille Paglia}

La intelectual italoamericana Camille Paglia ha desarrollado una teoría contra el feminismo que no deja de ser tendenciosa (Paglia, 2016, 2017 a, 2017 b). La argumentación de Paglia avanza por pasos bien definidos. El primer paso consiste en señalar que el feminismo y los estudios de género se han equivocado al abrazar el post-estructuralismo expresado en Foucault y otros, y al haber tomado distancia de la biología, de la arqueología, de la historia de la humanidad y de la antropología. El segundo paso es el señalar que hubo un tiempo en la historia de la humanidad en el que los hombres y las mujeres formaban grupos separados por una necesidad natural e histórica, y que las mujeres se encontraban felices en esa situación (permaneciendo en casa con otras mujeres, lavando la ropa y cuidando a los hijos). El tercer paso que da Paglia es señalas que hubo un momento (alrededor de los años 60) en el que las mujeres tuvieron la libertad sexual y pudieron salir a la esfera pública al igual que los varones. Pero, una vez conseguido eso, señala la intelectual, las mujeres de clase media alta y las mujeres de la clase trabajadora toman dos actitudes distintas frente al mundo y frente a los hombres. Las primeras buscan controlar al mundo y a los hombres para que la esfera pública sea segura y apacible como las salas de estar de sus casas. Las segundas, en cambio, se adaptan al mundo y aprenden a defenderse y a hablar de tal manera que su voz sea escuchada. Seguramente Paglia incluiría en el primer grupo a las mujeres del colectivo \#metoo y parte de los grupos feministas contemporáneos.

De esta manera señala lo siguiente

Apelo a un respeto mutuo entre los sexos, pero también apelo a que las chicas de clase media dejen de pedir protecciones especiales. Hay demasiadas quejas sobre el comportamiento de los hombres, cuando de hecho lo que muchas chicas blancas de clase media-alta llevan al mercado laboral es una cierta forma de hablar que está desconectada de la hostilidad del mundo. Las mujeres de la clase trabajadora ya vengan del campo o de la calle entienden los riesgos y los peligros del mundo. 
Es responsabilidad de las mujeres entender los peligros del mundo. No esperar que el mundo sea una extensión de sus cómodas salas de estar de clase media. Y creo que eso es lo que ha pasado, veo mujeres cuyas voces no son suficientemente fuertes. Las mujeres tienen que aprender a no tener vergüenza, a ser ruidosas y confrontar cuando eso pasa. Veo demasiados casos de mujeres que se sienten frágiles ante algo que les parece degradante y que simplemente lo aceptan. Y eso no es. La primera vez que sucede, te defiendes, le pones un freno. Lo confrontes personalmente, inmediatamente. Cada día tú tienes una responsabilidad de proteger tu propia dignidad, y no importa si te da vergüenza o crees que la situación es engorrosa. Protégete a ti misma (Paglia, 2017 b).

Y Paglia sigue diciendo:

Cuando mi generación ingresó a la universidad en 1964 las reglas residenciales estaban en pleno apogeo. Las chicas tenían que regresar de vuelta a sus dormitorios a las 11 de la noche y los hombres podían andar por todos lados toda la noche. Entonces fue mi generación de mujeres la que dijo 'queremos el mismo trato que los hombres, queremos igualdad y la libertad que los hombres disfrutan'. Y la universidad dijo 'no, el mundo es peligroso. debemos protegerlas. Debemos protegerlas de las violaciones'. Y nosotros dijimos: 'iDennos la libertad de arriesgar una violación!' . La libertad es el valor, no la protección. Me entristece ver a las jóvenes de hoy que quieren que vuelvan las figuras parentales, que quieren la vigilancia, que quieren salir corriendo a los comités cuando algo sale mal en una cita. Ellas tienen que gobernar sus relaciones con los hombres o con quien sea, y esa será la única forma en que las mujeres conseguirán ser libres y completamente iguales (Paglia, 2017 b).

En el argumento de Paglia podemos encontrar una direccionalidad conservadora respecto del feminismo y de las relaciones entre los sexos. En primer lugar, al rechazar el post-estructuralismo para basarme más bien en la biología, en cierto relato de la historia de la humanidad y en ciertos elementos antropológicos está descartando la manera en que los hombres y las mujeres se perciben a sí mismos, además del hecho de que las relaciones entre ambos sexos no han sido de igualdad, sino que ha estado inscrita en un sistema en el cual los hombres ejercían dominación sobre la mujer. La existencia de una sociedad androcéntrica, donde la misma historia y los mismos datos antropológicos y biológicos son interpretado desde una perspectiva masculina.

Por otro lado, ella afirma que en el mundo contemporáneo la esfera pública es igual de peligrosa para los hombres que para las mujeres, pero esa afirmación puede ser refutada fácilmente si se entiende que en sociedades centradas en los varones la esfera pública se encuentra articulada de tal manera que beneficie a los hombres y perjudique a las mujeres. La esfera pública es, indudablemente, hostil para las mujeres. El hecho de que las mujeres de la clase trabajadora hayan encontrado los recursos para defenderse no significa que ellas no se encuentren expuestas. De hecho, la explotación capitalista es más radical con ellas que 
con los varones. De otro lado surge la pregunta ¿por qué las mujeres de la clase trabajadora deben de hacer mayores esfuerzos que los varones para protegerse en la esfera pública? ¿Acaso porque como señala Paglia - simplemente es así? ¿Porque así es la realidad? O, más bien ¿Por qué el sistema social articulado a medida de los varones resulta ser injusto para con las mujeres?

Finalmente, el argumento de la intelectual italoamericana encuentra su vuelta de tuerca cuando señala que las mujeres tienen que entender que por su vestimenta podrían provocar la agresión de los varones, debido a la estructura biológica de los últimos. Dicho argumento se parece al del Cardenal Cipriani, que señala que las mujeres se exponen como en un escaparate y en vez de ser víctimas de las agresiones sexuales, son causantes de éstas. El argumento de Paglia se desliza en esa dirección, a saber, la de lanzar una acusación a las víctimas porque, supuestamente, "el mundo es así y las relaciones entre los sexos tiene esa realidad". De manera que, de acuerdo a Paglia, las mujeres deberían de dejar de acusar a los varones y entender cuál es la realidad del mundo contemporáneo.

\section{3.- Ocultando el marco neoliberal}

Las críticas de Deneuve, de Vargas Llosa y de Paglia terminan ocultando el marco neoliberal en el que se desarrolla la nueva explotación de la mujer. Pero, lo mismo ocurre con los grupos \#metoo y otros afines a ellos. Al centrar el debate sobre la cuestión del acoso, dejan fuera la crítica a la economía política. Ciertamente, el acoso y la violencia contra la mujer es un problema de primer orden respecto del cual hay que tomar medidas concretas. Pero si la crítica feminista termina centrándose sólo en ese aspecto, estaría atacando el problema de la opresión al que las mujeres se encuentran expuestas en las sociedades actuales, pero dejan fuera el problema de la explotación, con lo que la crítica feminista pierde potencial crítico y emancipador. En ese sentido, el debate en torno al acoso parece inscribirse en la agenda del feminismo de derecha que, como hemos visto, Rosa María Palacios podría perfectamente suscribir. La solución contra el mal del acoso es dotar a las mujeres de la educación suficiente y de los mecanismos mediáticos, sociales, legales y políticos para que puedan ponerle freno. Pero, sin una crítica adecuada del proyecto político y económico del neoliberalismo, la emancipación de la mujer va a encontrarse truncada. Es por ello que el feminismo debe revitalizar sus fuerzas progresistas para cuestionar al neoliberalismo.

Lo que debe quedarnos claro es que cuando hablamos del feminismo hoy en día es imposible pensarlo como un movimiento unitario y homogéneo. Como he mencionado, hay feminismos de derechas (en plural) y de izquierdas (también en plural). Una forma útil de ubicarse en medio de esta multiplicidad de posturas en el feminismo es verlas a través de los lentes del proceso del neoliberalismo, es decir, ver cuál es su posición respecto del proyecto político neoliberal que se encuentra en acción actualmente. A partir de este lente podemos ver que hay feminismos que se adaptan al neoliberalismo y otros que lo cuestionan. Aquellos que se adaptan, terminan siendo de derecha o realizando un giro hacia la derecha. Se trata de los feminismos enroscados en el concepto de "reconocimiento de la identidad". Estos feminismos han abandonado la lucha por la distribución justa y han apostado por exigir el reconocimiento de una identidad sustancializada (en torno a la 
idea de que ser mujer significa X, Y y Z). Dentro de este grupo se puede encontrar feministas que reivindican los valores tradicionales y que abogan por que la mujer se mantenga en condiciones de opresión, y otros que reivindican dotar de derechos y capacidades a las mujeres para que puedan defenderse de la opresión.

El feminismo en lucha sólo contra la opresión es compatible con las exigencias de los aglutinamientos Ni una menos y \#metoo. Aceptan la explotación, y rechazan la opresión y el abuso. No presentan una crítica clara contra el proyecto político neoliberal. En cambio, los feminismos progresistas se dirigen a cuestionar el sistema económico. Pero en estos feminismos de izquierda hay quienes no han realizado aún un claro análisis del neoliberalismo y apuntan a romper el "techo de cristal" pero tienen una ceguera respecto al "techo de hierro" que impone el proyecto neoliberal y que incrementa la desigualdad socioeconómica entre mujer y mujer. Este feminismo de izquierda apunta hacia la distribución, pero no apunta con claridad a criticar el sistema que impide en marco político democrático. En cambio, hay un segundo feminismo de izquierda que apuntala contra el neoliberalismo y apuesta por el reconocimiento de status, la redistribución socioeconómica y la paridad participativa a nivel político, de manera tal que enarbola la bandera de lo que Fraser denomina democracia radical. Esta segunda forma del feminismo de izquierda tiene un adecuado diagnóstico de las relaciones de reconocimiento, económicas y políticas surgidas con la revolución neoliberal. Si bien, dentro de estos grupos que he esbozado, es posible encontrar variaciones internas respecto de diferentes posturas y de diferentes análisis respecto de quien gana y de quien pierde en cada propuesta, nos inclinamos a aceptar la propuesta del feminismo asociada a la democracia radical defendida por Nancy Fraser.

\section{BIBLIOGRAFÍA}

BANSINATH, Bindu (2018). El dolor de descubrir que eres Lolita. En: https://www.nytimes. com/es/2018/02/16/modern-lovelolita-tio-abuso/.

CIPRIANI, Juan Luis (2017), declaraciones. En: https:// w w w. y o u t u b e.co m / watch?v=F53ZjWJrZNg.

DE HAAS, Caroline (2017). Respuesta al Manifiesto de las intelectuales francesas contra \# metoo. En: https://www. francetvinfo.fr/societe/droitsdes-femmes/tribune-les-porcset-leurs-allie-e-s-ont-raisonde-sinquieter-caroline-de-haaset-des-militantes-feministesrepondent-a-la-tribune-publieedans-le-monde_2553497.html.

DENEUVE, Katherine (2017). Manifiesto de las intelectuales francesas contra \#metoo. En: https://www.infobae.com/america/ mundo/2018/01/09/el-manifiestocompleto-de-las-intelectualesfrancesas-contra-el-metoo/.

FOUCAULT, Michel (2008). Nacimiento de la biopolítica: curso en el College de France (19781979), México: FCE.

FRASER, Nancy (2008). Escalas de justicia, Barcelona: Herder.

FRASER, Nancy (20015). Fortunas del feminismo, Quito: Traficantes de sueños. 
HONNETH, Axel (2009). Crítica del agravio moral. Patologías de la sociedad contemporánea. México: FCE.

MENGOLINI, julia (2018). ¿Puede el feminismo se de derecha? En: http://artepolitica.com/articulos/ puede-el-feminismo-ser-dederecha/.

NUSSBAUM, Martha (1999). Sex and social justice, New York: Oxford University Press.

NUSSBAUM, Martha (2000 a). Las mujeres y el desarrollo humano, Barcelona: Herder.

NUSSBAUM, Martha (2000 b). The Professor of Parody en: TheNewRepublic.com.

PAGLIA, Camille (2017 a). Mujeres libres, hombres libres. Primera parte. En:https://www.youtube. com/watch?v=PA-N8U-bHfg,
PAGLIA, Camille (2017 b). Mujeres libres, hombres libres. Segunda parte. En: https://www.youtube. com/watch?v=CFYL_p86r3c,

PAGLIA, Camille (2016) El feminismo de hoy no es feminismo. En: https://www.youtube.com/ watch?v=Oaf7oPoxJV8

PALACIOS, Rosa María (2018). Sin Guión del 8 de marzo del 2018. En: https://www.youtube.com/ watch?v $=g \mathrm{H}-\mathrm{uvXL7H2E}$.

REYES, Ronald (2018). Por qué el populismo no es democrático. En: http://ronrloayza.blogspot. pe/2018/04/por-que-el-populismono-es-democratico.html.

VARGAS LLOSA, Mario (2018). Muevas inquisiciones. En: https:// elpais.com/elpais/2018/03/16/ opinion/1521215265_029385.html. 